\title{
Elevation of Serum CA-125 in Mucinous Cystadenoma of the Ovary with Torsion: A Rare Occurrence and Review of the Literature
}

\author{
Tadao Okada ${ }^{1}$, Shohei Honda ${ }^{1}$, Hisayuki Miyagi ${ }^{1}$, Masashi Minato ${ }^{1}$, \\ Kanako C. Kubota ${ }^{2}$, Akinobu Taketomi ${ }^{3}$ \\ ${ }^{1}$ Department of Pediatric Surgery, Hokkaido University Hospital, Sapporo, Japan \\ ${ }^{2}$ Department of Surgical Pathology, Hokkaido University Hospital, Sapporo, Japan \\ ${ }^{3}$ Department of Gastroenterological Surgery I, Hokkaido University \\ Graduate School of Medicine, Sapporo, Japan \\ E-mail:okadata@med.hokudai.ac.jp \\ Received May 23, 2011; revised August 14, 2011; accepted October 17, 2011
}

\begin{abstract}
Aim: Benign neoplasms of the ovary originating from epithelial tissue are common tumors in adult women. They are, however, rarely seen in children or adolescent girls with elevation of serum carbohydrate antigen125 (CA-125). The present report describes a rare case of premenarchal women with a giant mucinous cystadenoma (MCA) of the ovary with torsion complicated with elevation of serum CA-125. Case: A 15-yearold, premenarchal, previously healthy girl was referred to our hospital with a 2-week history of left lower abdominal pain. Physical examinations showed a firm and mobile mass with tenderness in the left lower quadrant. Tumor markers showed CA-125 at $124.1 \mathrm{U} / \mathrm{ml}$. An enhanced computed tomography scan showed a multiloculated tumor that was partly solid, compressing the small intestine, uterus, and urinary bladder, but no signs of organ invasion, lymph node swelling, or ascitis. Via a lower transverse incision, the right Fallopian tube was observed to have twisted $1620^{\circ}$ counterclockwise, and a tense cyst measuring $22.0 \times 12.0 \times$ $10.5 \mathrm{~cm}$ and weighing $1.78 \mathrm{~kg}$ was found in the right ovary. Release from torsion and unilateral salpingooophorectomy with tumor removal was performed because blood flow to the right Fallopian tube did not improve after torsion release. The histopathological findings showed an MCA of the ovary without cell dysplasia. The patient did not receive adjuvant chemotherapy following surgery. There was no evidence of recurrence at 2 years. Disucussion: We need to consider MCA of the ovary when there is elevation of the serum CA-125 and an ovarian mass.
\end{abstract}

Keywords: CA-125, Mucinous Cystadenoma of the Ovary, Salpingo-Oophorectomy, Torsion

\section{Introduction}

Benign neoplasms of the ovary originating from epithetlial tissue are common tumors in adult women. They are, however, rarely seen in children or adolescent girls. $\mathrm{Mu}-$ cinous tumors of the ovary occur principally in middle adult life and are rare prior to menarche [1]. Such tumors can be classified as adenoma, borderline malignancy, and adenocarcinoma according to cytological and structural atypia [2]. Mucinous cystadenoma (MCA) appears as a large cystic mass and is often multiloculated and contains sticky gelatinous fluid. T. Sri Paran et al. described only 13 previous cases of benign MCA of the ovary in perimenarchal girls reported in the literature in 2006 [1]. Especially, giant MCA of the ovary with torsion complicated with elevation of serum cancer antigen-125 (CA125 ) is extremely rare in pediatrics.

The present report describes the very rare case of a 15year-old girl with giant MCA of the ovary with torsion complicated with the elevation of serum CA-125, and successful surgical treatment. We review the literature on this type of relationship between MCA of the ovary with torsion and the elevation of serum CA125, and discuss the clinical features of this complication. Furthermore, the possible cause of torsion in MCA of the ovary in premenarchal women was explored in this report. 


\section{Case Report}

A 15-year-old, premenarchal, previously healthy girl was referred to our hospital with a 2-week history of left lower abdominal pain. Physical examinations showed a firm and mobile mass with tenderness in the left lower quadrant. Tumor markers showed $\alpha$-fetoprotein at $2.3 \mathrm{ng} / \mathrm{ml}$, $\beta$-hCG $<0.5 \mathrm{ng} / \mathrm{ml}$, and CA-125 at $124.1 \mathrm{U} / \mathrm{ml}$ (normal: 2.4 - 36.3).

An enhanced computed tomography (CT) scan showed a multiloculated tumor that was partly solid, compressing the small intestine, uterus, and urinary bladder, but no signs of organ invasion, lymph node swelling, or ascitis (Figure 1). On the basis of these findings and physical signs, an ovarian tumor with torsion was suspected, and emergent surgical intervention was scheduled.

Via a lower transverse incision, the right Fallopian tube was observed to have twisted $1620^{\circ}$ counterclockwise, and a tense cyst measuring $22.0 \times 12.0 \times 10.5 \mathrm{~cm}$ and weighing $1.78 \mathrm{~kg}$ was found in the right ovary (Fig- ure 2). The left ovary was normal. Release from torsion and unilateral salpingo-oophorectomy with tumor removal was performed because blood flow to the right Fallopian tube did not improve after torsion release. Most of the tumor was necrotic, and congestive. A viable part of the tumor, histologically, multilocular cystic neoplasm was lined by a single layer of columnar mucinous cells with small basilar nuclei. There were no signs of epithelial atypia or intraepithelial carcinoma in the epithelium lining the cyst surface. The histopathological findings showed an MCA of the ovary without cell dysplasia. The patient did not receive adjuvant chemotherapy following surgery. There was no evidence of recurrence at 2 years.

\section{Discussion}

The incidence of combined benign and malignant ovarian tumors has been estimated to be around 2.6 cases per 100,000 in girls younger than 15 years of age [1]. They

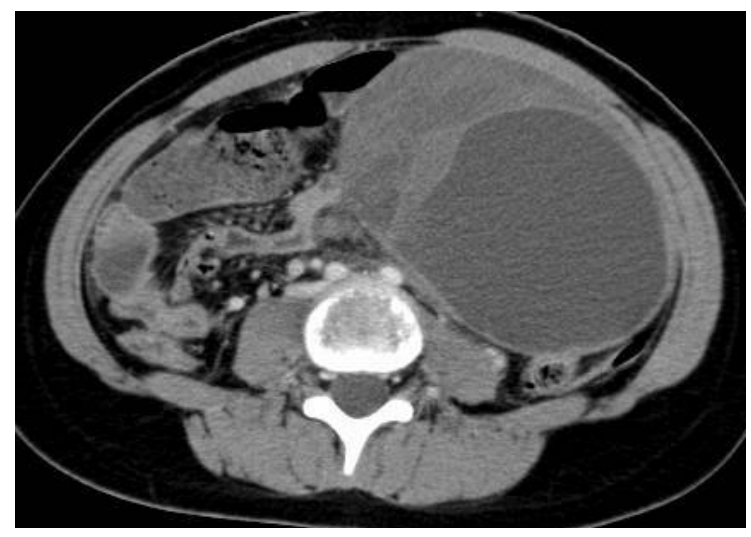

(a)

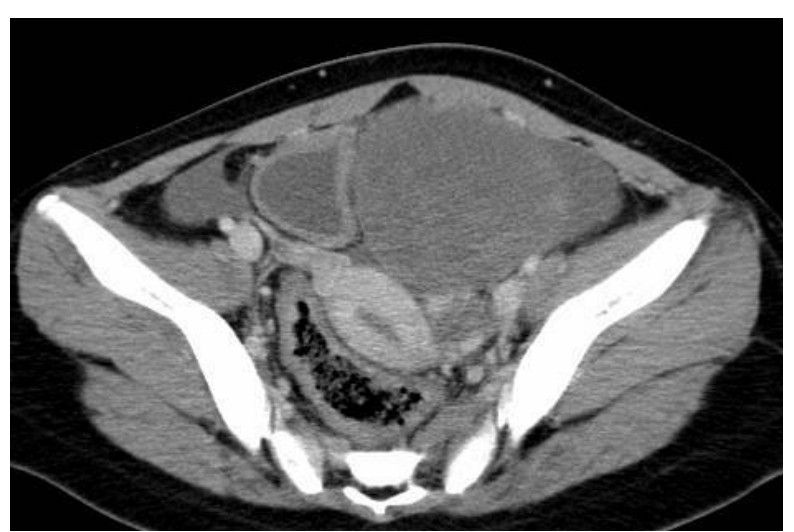

(b)

Figure 1. (a) Enhanced CT scan showed a multiloculated tumor that was partly solid; (b) The tumor compressed the small intestine, uterus (arrow), and urinary bladder (arrowhead), but no signs of organ invasion, lymph node swelling, or ascitis.
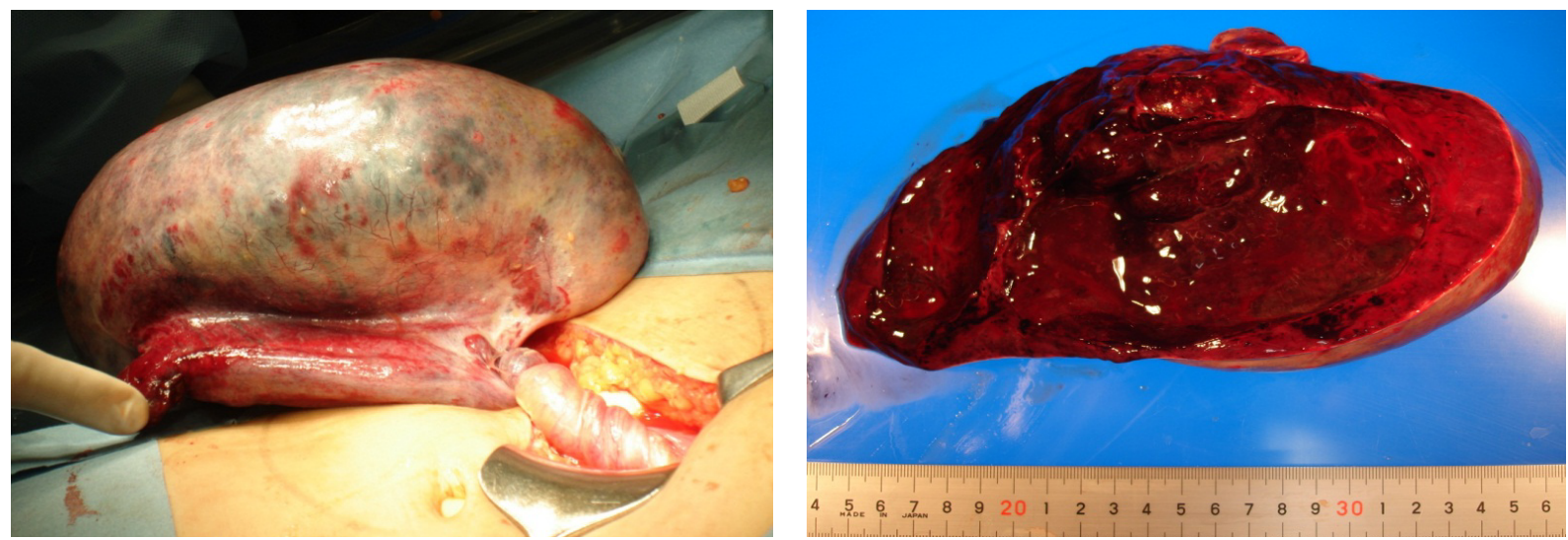

Figure 2. Intraoperative and transectional macroscopic findings. Via a lower transverse incision, the right Fallopian tube was observed to have twisted $1620^{\circ}$ counterclockwise, and a tense cyst measuring $22.0 \times 12.0 \times 10.5 \mathrm{~cm}$ and weighing $1.78 \mathrm{~kg}$ was found in the right ovary; (b) Most of the tumor was necrotic and a part was solid. 
represent less than $2 \%$ of all tumors in girls below 16 years of age. Less than $20 \%$ of ovarian malignancies reportedly arise from ovarian surface epithelial cells [3]. Epithelial tumors of the ovary are rare prior to puberty [4].

The differential diagnosis of ovarian masses in adolescents includes cyst formation, torsion with consecutive edema, benign or malignant ovarian neoplasm, and involvement of the ovary in lymphoma, leukemia, or metastatic disease [5]. Deprest et al. reviewed 1700 reported cases of ovarian neoplasms in females under 20 years of age, and reported epithelial tumors in $17 \%$, of which $26 \%$ were malignant [6]. Ovarian MCA is an epithelial tumor, it is usually unilateral, and only $10 \%$ of cases, are malignant [7]. They occur principally in middle adult life and are exceptional in children and adolescents, such as the present case [5].

CA-125 is a glycoprotein that is produced by certain tumors, such as malignant epithelial ovarian tumors. In premenopausal patients, benign findings such as leiomyomas, endometriosis, menstruation, and pregnancy may elevate CA-125. Paran et al. reported that CA-125 was within the normal range in 6 perimenarchal girls with ovarain MCA [1]. Especially, MCA of the ovary with torsion complicated with the elevation of serum CA-125 is an extremely rare occurrence in pediatrics.

K-ras mutations are common in mucinous ovarian tumors, are probably an early event in mucinous ovarian tumors, and are probably an early event in mucinous ovarian carcinogenesis [2]. Interestingly, some microdissected mucinous tumors had the same K-ras mutation in histologically benign, borderline, and malignant areas of the same tumor [8]. Unfortunately, we did not examine K-ras mutation.

In young patients with apparently benign ovarian cysts requiring removal, the conservative approach of ovarian cystectomy is advocated to enable the retention of functioning ovarian tissue for endogenous hormone production and future conception [4]. However, when a huge cyst is encountered such as in our case, preserving the ovarian tissue might be difficult. In the present case, it was not possible to determine the origin of the mass before laparotomy, and there were no findings ruling out malignancy because of the solid component of the tumor and the elevation of serum CA-125. Therefore, the treatment of choice was complete resection rather than biopsy. Conservative surgery (i.e., unilateral salpingo-oophorectomy) is generally sufficient for benign lesions and early-stage ovarian tumors of borderline malignancy [9].

Torsion of MCA such as that reported here is rarely seen in children or adolescent girls. Ovarian tumors that undergo torsion can mimic acute appendicitis and may lead to emergency surgery [10].

\section{Conclusions}

In conclusion, we need to consider MCA in adolescent girls with the elevation of serum CA-125 and the presence of a very large ovarian mass.

\section{References}

[1] T. S. Paran, A. Mortell, D. Devaney, A. Pinter and P. Puri, "Mucinous Cystadenoma of the Ovary in Perimenarchal Girls," Pediatric Surgery International, Vol. 22, No. 3, 2006, pp. 224-227. doi:10.1007/s00383-005-1624-1

[2] W. R. Hart, "Mucinous Tumors of the Ovary: A Review," International Journal of Gynecology and Pathology, Vol. 24, No. 1, 2005, pp. 24-25.

[3] J. L. Breen and W. S. Maxson, "Ovarian Tumors in Children and Adolescents," Clinical Obstetrics and Gynecology, Vol. 20, No. 3, 1977, pp. 607-623. doi:10.1097/00003081-197709000-00010

[4] B. Baksu, A. Akyol, I. Davas, A. Yazqan, J. Ozqul and C. Tanik, "Recurrent Mucinous Cystadenoma in a 20-Yearold Woman was Hystectomy Inevitable?" Journal of $\mathrm{Ob}$ stetrics and Gynaecology Research, Vol. 32, No. 6, 2006, pp. 615-618. doi:10.1111/j.1447-0756.2006.00453.x

[5] C. Flotho, K. Rückauer, U. Duffner, E. Berqstässer, N. Bohm and C. M. Niemeyer, " Mucinous Cystadenoma of the Ovary in a 15-Year-Old Girl," Journal of Pediatric Surgery, Vol. 36, No. 6, 2001, p. E6. doi:10.1053/jpsu.2001.24019

[6] J. Deprest, P. Moerman, P. Corneillie, P. Ide, "Ovarian Borderline Mucinous Tumor in a Premenarchal Girl: Review on Ovarian Epithelia Cancer in Young Gilrs, Gynecologic Oncolology, Vol. 45, No. 2, 1992, pp. 219-224.

[7] E. E. Lack, R. H. Young, R. E. Scully, "Pathology of Ovarian Neoplasms in Childhood and Adolescence," $\mathrm{Pa}$ thology Annual, Vol. 27, 1992, pp. 281-356.

[8] M. Mandai, I. Konishi, H. Kuroda, T. Komatsu, S. Yamamoto, K. Nanbu, K. Matsushita, M. Fukumoto, H. Yamabe and T. Mori, "Heterogenous Distribution of K-rasmutated Epithelia in Mucinous Ovarian Tumors with Special Reference to Histopathology," Humman Pathology, Vol. 28, No. 1, 1998, pp. 34-40. doi:10.1016/S0046-8177(98)90387-2

[9] C. J. Link Jr., E. Reed, G. Sarosy and E. C. Kohn, "Borderline Ovarian Tumors," American Journal of Medicine, Vol. 101, No. 2, 1996, 217-225. doi:10.1016/S0002-9343(96)80079-9

[10] P. W. Cronen and H. S. Nagaraj, "Ovarian Tumors in Children," Southern Medical Journal, Vol. 81, No. 4, 1988, 464-468. doi:10.1097/00007611-198804000-00014 